\title{
Evidence-Based Tools for Dietary Assessments in Nutrition Epidemiology Studies for Dementia Prevention
}

\author{
K.A. Abbott' , J.M. Posma 2 , I. Garcia-Perez ${ }^{2}$, C. Udeh-Momoh ${ }^{1}$, S. Ahmadi-Abhari ${ }^{1}$, L. Middleton ${ }^{1,3}$, G. Frost ${ }^{2}$

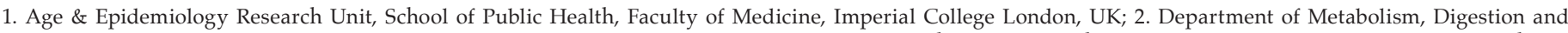

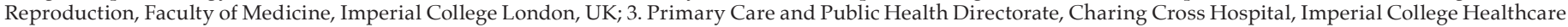
NHS Trust, UK

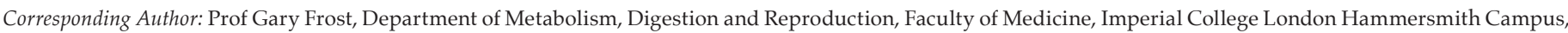
Commonwealth Building, Du Cane Road, London W12 ONN, United Kingdom, Tel: +44 (0)20 7594 0959. Fax: +44 (0208383 8320, Email : g.frost@imperial.ac.uk

\begin{abstract}
Increasing evidence proposes diet as a notable modifiable factor and viable target for the reduction of Alzheimer's Disease risk and age-related cognitive decline. However, assessment of dietary exposures is challenged by dietary capture methods that are prone to misreporting and measurement errors. The utility of -omics technologies for the evaluation of dietary exposures has the potential to improve reliability and offer new insights to pre-disease indicators and preventive targets in cognitive aging and dementia. In this review, we present a focused overview of metabolomics as a validation tool and framework for investigating the immediate or cumulative effects of diet on cognitive health.
\end{abstract}

Key words: Metabolomics, dietary assessments, nutrition, nutriome, Alzheimer's disease, dementia, evidence-based tools, precision nutrition.

\section{Introduction}

A steady but certain shift towards the integration of evidence-based tools to enhance traditional methods of dietary assessment is currently emerging in the field of nutrition science. This is noteworthy, because much of our understanding of dietary associations in relation to Alzheimer's disease (AD) and other late onset dementia (LOD) forms has derived from self-administered dietary assessments which are prone to biases and reporting errors.

Specifically, the integration of -omics technologies into the evaluation of dietary exposures heralds a new era of research that can be more robust with potential to improve reliability and offer novel insights to pre-disease indicators and preventive targets in cognitive aging and dementia.

Recent years saw a plethora of publications from non-pharmacological interventional studies such as the FINGER randomized controlled trial (RCT) providing evidence that diet, as part of a multi-domain lifestyle intervention, can contribute to significantly mitigate decline in cognitive performance and potentially delay the onset or progression of $\mathrm{AD}$ and other types of dementia (1). These promising results, based on simultaneous management of several vascular and lifestyle related risk factors, in which the intervention group were assigned a multidomain intervention consisting of diet, exercise, cognitive training, vascular risk monitoring versus the control group assigned general health advice, showed efficacy after a 2 year period in improving cognitive performance in at-risk, but cognitively unimpaired and/or mildly impaired individuals (2). This was pivotal because interest in lifestyle modifications to reduce disease prevalence was affirmed and personalised strategies/optimal timing became discussion points.

The increasing awareness that pathological changes in AD-LODs develop many years prior to clinical disease onset and the results of several observational and interventional studies has led the field to acknowledge that the pre-clinical or early clinical stages are the optimal time points for intervention; thus, attention has shifted towards health-promoting behaviours such as healthy diet, maintaining an optimal weight, physical and mental activities and social interactions, in addition to potential pharmacological therapies, if and when novel disease modifying medicines of proven efficacy and cost effectiveness become available.

Diet and nutrition are viable targets for strategies aimed at preserving cognitive health in older adults and have become a focus in dementia related studies. Moreover, there has been a gradual shift from single nutrient analyses towards dietary pattern analyses, reflecting trends in nutritional epidemiology, in which synergistic effects of food combinations and possible nutrient interactions are deemed more informative (3).

Despite accruing evidence from a wealth of epidemiological studies, showing that adherence to healthy dietary patterns, such as the Mediterranean, Nordic, DASH, MIND or anti-inflammatory diets may lend neuroprotective effects, and more recently, the ketogenic diet, in its capacity to alter brain metabolism (4-7), these findings have not translated uniformly into dietary guidelines, to improve cognitive health and disease burden reduction in older adults, due to 
Figure 1. From urine collection to dietary assessment pipeline

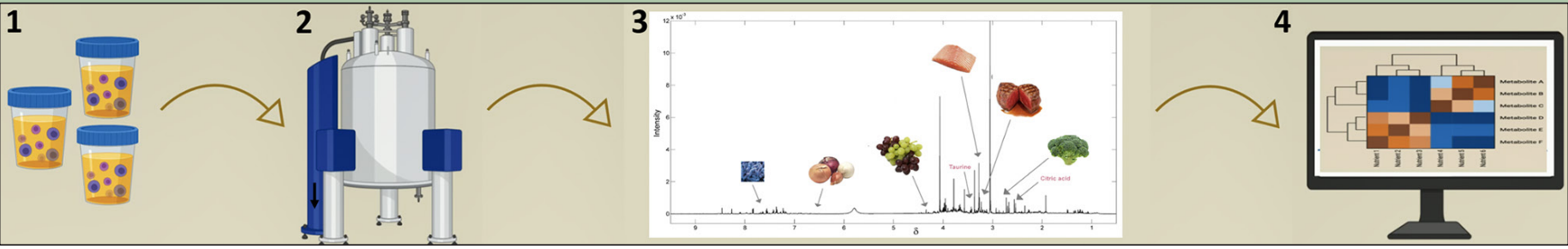

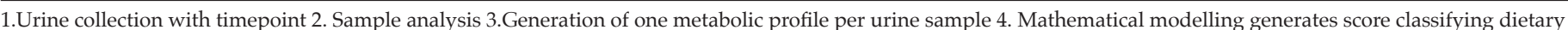
profile in relation to the adherence to dietary recommendations

inconsistencies in the literature.

In order to understand the precise effects of dietary intake on cognitive function and their mechanism of action, dietary assessment methodologies must be able to measure dietary intake as accurately as possible. As it stands, current methods of dietary assessment typically collect self-reported data through food-frequency questionnaire (FFQ), 24-hour recalls, or food diaries which are dependent on subject recall and cognitive functioning; all self-reported methodologies have considerable scope for measurement error and inherent bias, with under-reporting biased towards unhealthy foods and dietary energy intake and over-reporting towards healthier foods (8).

Also, there is potential error in estimating portion sizes and the limitation of the number of foods and dishes that have been directly analysed. Reporting is known to deteriorate further in the obese and likely to include significant inaccuracies in older populations. Prevalence of misreporting has been estimated as between $30-88 \%$ in epidemiological surveys (9). In large scale studies, use of FFQ's are commonly applied, but since these are tailored to suit the population they serve, there is wide variation in number and range of items appearing on food lists.

Another limiting factor with traditional dietary assessment models is the limited number of nutrients that have been measured accurately in food. Evidence suggests that bioactive molecules in food such as polyphenols may play a role, but these molecules are not measured in most nutritional data sets. This limits the scope of understanding between the chemical composition of food and cognitive associations.

Reliability of dietary assessment is further compromised by analytical methods applying either a priori methodologies using constructed scores based on an underlying hypothesis and dietary guidelines which do not reflect entire dietary intake or a posteriori methods, applying data reduction techniques, such as principal component analysis, factor analysis, or cluster analysis to categorise on intercorrelations which provides insight to shared characteristics within a population but has limited comparability and reproducibility in other population samples. Both approaches are limited by pre-defined selections of food and nutrient groupings resulting in varying interpretations of dietary exposures and disease risk.
In recent years, the advent of online dietary assessment tools has made data collection and analysis much easier, albeit not mitigating the biases, as outlined above; also including technological competence in older adults, recently noted as a possible confounder for reporting accuracy; a feasibility study on use of online dietary recalls among older adults indicated that participants who completed multiple recalls reported higher selfconfidence with technology and a higher technology readiness score than those who did not complete any recalls (10). There is, still, a need for evidence-based tools to be evaluated for validation and reliability in study outcomes and clarity in mechanisms that might be protective against dementia. We therefore propose a focus on applying metabolomics as a validation tool and framework for investigating the immediate or cumulative effects of diet on cognitive status and decline.

\section{Metabolomics as a validation tool}

The implementation of high-throughput -omics technologies in dietary assessments holds promise for evidence-based data, by providing objective measures of dietary intake in targeted or untargeted analyses, thus mitigating the risks of bias and subjectivity of selfadministered data collecting methods.

Such technologies include Nuclear Magnetic Resonance (NMR) spectroscopy and Mass Spectroscopy (MS) based techniques using Liquid Chromatography (LC-MS) and Gas Chromatography (GC-MS) for improved separation.

A novel approach, developed to assess dietary intake against metabolic profiles, has been the adoption of NMR spectroscopy in urinary analyses to detect concentrations of small molecule metabolites which reflect "actual" rather than "estimate" measures of food intake. In this way, distinguishable metabolites can be used to validate dietary intake of FFQs and dietary patterns (8).

For example, a higher intake of fish consumption or protein in a FFQ can be validated by protein related metabolites in the metabolomic profile or other metabolites such as proline betaine, a marker of citrus fruit intake (11). Established dietary biomarkers, such as urinary sodium or nitrogen balance can track intake of specific nutrients, however metabolic profiling goes further in providing insight to metabolomic response of 


\begin{tabular}{|c|c|c|c|c|c|}
\hline Author & Study design & Size & Age & Dietary pattern groups & Metabolites \\
\hline Heinzmann et al, 2010 & $\mathrm{RCT}$ & 8 & $28-45$ & $\begin{array}{l}\text { Supplementary mixed-fruit meal with standard } \\
\text { dinner vs standard dinner }\end{array}$ & $\begin{array}{l}\text { Proline betaine a putative biomarker of citrus } \\
\text { consumption }\end{array}$ \\
\hline Garcia-Perez et al, 2015 & $\begin{array}{l}\text { Randomized } \\
\text { crossover }\end{array}$ & 25 & 21-65 & $\begin{array}{l}4 \text { diets varying in compliance to WHO eating } \\
\text { guidelines }\end{array}$ & $\begin{array}{l}\text { Diet 1: healthier food biomarkers: hippurate } \\
\text { (marker of fruit and vegetable), N-acetyl- } \\
\text { S-methyl-cysteine-sulfoxide (cruciferous } \\
\text { vegetables), dimethylamine, and TMAO (fish), } \\
\text { and 1-methylhistidine and 3-methylhistidine } \\
\text { (oily fish and chicken) } \\
\text { Diet 4: Higher concentration of nine urine } \\
\text { metabolites associated with higher red meat } \\
\text { quantity (O-acetylcarnitine, carnitine, and } \\
\text { creatine) and sugars }\end{array}$ \\
\hline Loo et al, 2017 & RCT (OmniHeart) & 158 & $30-80$ & OmniCarb diet, OmniProt diet; OmniMFA diet & $\begin{array}{l}\text { Increased secretion of carnitine and creatine } \\
\text { with OmniProt - (higher protein diet) }\end{array}$ \\
\hline
\end{tabular}

overall dietary intake following digestion, absorption and metabolism, providing insight to functional relationships between diet and health outcomes (12).

In addition to the feasibility of using metabolic profiles to validate dietary patterns, merging -omics with dietary assessments provides an opportunity to monitor and objectively assess dietary intake against healthy eating targets using urine composition; thus, enabling the quantification and monitoring of the potential effect of the adherence to or changes in dietary pattern in response to risk reduction strategies.

Several nutrition studies have explored how metabolomics can establish accurate associations between diet and disease risk to predict health outcomes. In a randomised, controlled trial, four dietary patterns, administered in controlled feeding conditions, revealed diet-discriminatory metabolomic profiles associated with different degrees of non-communicable disease risk, based on compliance to the WHO recommended healthy diets (8). The model was validated using internal and external cohort data with 24-hour recalls and found to associate with predicted scores of dietary profiles derived from the urinary metabolic profiles.

Applying the same methodology, a recent study demonstrated agreement between urinary metabolic biomarkers and self-reported data built on a model of 46 urinary metabolites, paired with 24-hour dietary recalls from 1,848 US individuals to accurately differentiate between healthy and unhealthy dietary patterns (13). Additionally, in a RCT of subjects with hypertension, the presence of specific metabolic markers suggested a mediating response to pre-disease markers in the gut microbiome (14).

Furthermore, an integrated metabolomic approach across platforms (NMR, LC-MS and GC-MS) may allow to accurately assess thousands of molecules associated with food intake that are not captured in current food tables.

The integration of nutritional metabolomics into dementia research invites new possibilities; improved reliability in dietary assessments might prove valuable but may only be the tip of the iceberg. If we consider that the 'functional nutriome', used to describe "chemically-defined diet-derived molecular species" (13) and expression of metabolic phenotype, may affect diet and disease risk, this has relevance in the context of $\mathrm{AD}$ risk and precision nutrition. We know that multiple processes leading to cognitive decline and dementia begin many years prior to the onset of cognitive decline; in determining whether there are specific metabolomic markers present in the earliest stage of cognitive decline which are observed in the food-derived metabolome, would be an important step in identifying predictive signatures of disease risk for earlier diagnosis in AD and other LODs. Furthermore, metabolite profiles can differentiate dietary patterns, so theoretically diet can be interrogated without dependency on self-reported dietary assessments.

\section{Biofluid used for metabolomic dietary assessment}

The vast majority of current metabolomic-based dietary assessment use either plasma or urine, however evidence on comparability of the two biofluids in relation to diet is limited. For urine, protocols are beginning to emerge which give guidance on the use of spot samples. Evidence suggests the first void urine gives the most relevant information (15). Moreover, the application of metabolomics to measurement molecular profile of food allows molecules to be traced from consumption to metabolism endpoints.

However, a major limitation of current metabolomic dietary assessments is that it is not possible to assess carbohydrate intake fully, due to the lack of relevant biomarkers. Given that carbohydrates are a major source of energy in most diets this is a limitation and an area of active research. Another limitation relates to the timeframe of the biofluid sample report; since it has been suggested that the first void urine relates to the previous day's dietary intake (16). 


\section{Dementia-related metabolomic studies}

The National Institute on Aging-Alzheimer's Association (NIA-AA) research guidelines for AD and cognitive decline due to AD pathology, emphasize the need for the implementation of biochemical markers and validation measures to unify findings across the variation of diverse methodologies (17), under the revised NIAAA Research Framework biomarker-based AD criteria for research purposes of amyloid and tau abnormal accumulation and neurodegeneration $\mathrm{AT}(\mathrm{N})$ (18).

The advancement of AD biomarkers has gained momentum in recent years in determining the AT(N) status in vivo, through the use of positron emission tomography (PET), cerebrospinal fluid (CSF), plasmabased assays and magnetic resonance imaging (MRI) studies, as core indicators of disease pathology (19). Moreover, metabolites in different biofluids (serum, plasma, CSF, urine) are proving to be promising indicators of alterations in lipids, amino acids, hormones and other circulating metabolites and their potential associations with cognitive performance change (20), (21) and as precision medicine tools contributing to the classification of patients into cognitive status subgroups based on metabolite signatures (22).

However, the literature is lacking examples of diet-dementia related studies and reference models applying nutritional metabolomics in cognitive and dietary pattern associations. Results from one casecontrol study linked a baseline serum signature of 22 metabolites with subsequent cognitive decline over 12 years and suggested specific foods (coffee, cocoa and fish) may be protective (23). Despite the novelty of the findings, the study was not validated in other cohorts, so reproducibility is still unclear, delineating the need for additional high-quality studies that apply complementary metabolomic platforms and approaches to identity predictive signatures of $\mathrm{AD}$ risk and preventive targets in cognitive decline and dementia.

To conclude, the emergence of improved measurements of ageing and dietary biomarkers represents a potentially exciting new development in dementia research. The enrichment of dietary assessments through the application of novel -omics technologies provides an opportunity for greater confidence and reliability in self-reported measures, whilst allowing to better understand the metabolomic responses in relation to dietary and cognitive associations. As Alzheimer's and other late onset dementia forms are multi-faceted diseases, evidence-based and multi-pronged approaches are required in disentangling the respective roles of modifiable factors in disease development. It is our view that future research aimed at exploring the role of diet in brain health and dementia prevention should leverage emerging innovative high-throughput technologies, such as metabolomics, that may more sensitively and accurately inform on the functional nutriome.
Conflict of Interest: L T Middleton has received research funding (to Institution) from Janssen, Merck (USA), Gates, Takeda/ Millenium, Novartis, Invincro and EIT Health, outside the submitted work. S Ahmadi Abhari and L T Middleton were supported by the European Institute of Innovation and Technology (EIT) Health for the brain ageing PhD school. J M Posma received a Medical Research Council (MRC) funded HDR UK Rutherford Fund Fellowship (MR/S004033/1). Isabel Garcia-Perez received a NIHR Career Development Research Fellowships (NIHR-CDF-2017-10-032). J M Posma, I Garcia-Perez and G Frost hold a patent in preparation (GB2111739.5) on NMR spectrum data fitting. G Frost - has received research funding (to Imperial College) from Nestle, Unilever, Quorn and Heptaris. G Frost and I Garcia-Perez are directors of Melico Ltd a dietary metabolomic spinout company.

Open Access: This article is distributed under the terms of the Creative Commons Attribution 4.0 International License (http://creativecommons.org/ licenses/by/4.0/), which permits use, duplication, adaptation, distribution and reproduction in any medium or format, as long as you give appropriate credit to the original author(s) and the source, provide a link to the Creative Commons license and indicate if changes were made.

\section{References}

1. Kivipelto M, Mangialasche F, Ngandu T. Lifestyle interventions to prevent cognitive impairment, dementia and Alzheimer disease. Nature reviews Neurology. 2018;14(11):653. https:/ / doi.org/10.1038/s41582-018-0070-3

2. Ngandu T, Lehtisalo J, Solomon A, Levälahti E, Ahtiluoto S, Antikainen R, et al. A 2 year multidomain intervention of diet, exercise, cognitive training, and vascular risk monitoring versus control to prevent cognitive decline in at-risk elderly people (FINGER): a randomised controlled trial. The Lancet. 2015;385(9984):2255-63. https: / / doi.org/10.1016/S0140-6736(15)60461-5

3. $\mathrm{Fb} \mathrm{H}$. Dietary pattern analysis: a new direction in nutritional epidemiology. Current opinion in lipidology. 2002;13(1):3-9. https:/ / doi org / 10.1097/00041433-200202000-00002

4. Samadi M, Moradi S, Moradinazar M, Mostafai R, Pasdar Y. Dietary pattern in relation to the risk of Alzheimer's disease: a systematic review. Neurological Sciences. 2019;40(10):2031-43. https: / / doi.org/10.1007/s10072-019-03976-3

5. Chen X, Maguire B, Brodaty H, O'Leary F. Dietary Patterns and Cognitive Health in Older Adults: A Systematic Review. J Alzheimers Dis. 2019;67(2):583619. https: / / doi.org/10.3233/JAD-180468

6. Davis JJ, Fournakis N, Ellison J. Ketogenic Diet for the Treatment and Prevention of Dementia: A Review. Journal of Geriatric Psychiatry and Neurology. 2021;34(1):3-10. https:/ / doi.org/10.1177/0891988720901785

7. Behnaz S, Debora R, Susanna CL, Gerd F-I, Laura F, Wei-Li X. The Nordic Prudent Diet Reduces Risk of Cognitive Decline in the Swedish Older Adults: A Population-Based Cohort Study. Nutrients. 2018;10(2):229. https://doi. org/10.3390/nu10020229

8. Garcia-Perez I, Posma JM, Gibson R, Chambers ES, Hansen TH, Vestergaard $\mathrm{H}$, et al. Objective assessment of dietary patterns by use of metabolic phenotyping: a randomised, controlled, crossover trial. The Lancet Diabetes \& Endocrinology. 2017;5(3):184-95. https://doi.org/10.1016/s22138587(16)30419-3

9. Rennie KL, Coward A, Jebb SA. Estimating under-reporting of energy intake in dietary surveys using an individualised method. Br J Nutr. 2007;97(6):116976. https: / / doi.org/10.1017/ s0007114507433086

10. Ward HA, McLellan H, Udeh-Momoh C, Giannakopoulou P, Robb C, Wark PA, et al. Use of Online Dietary Recalls among Older UK Adults: A Feasibility Study of an Online Dietary Assessment Tool. Nutrients. 2019;11(7):1451. https: / / doi.org/10.3390/nu11071451

11. Heinzmann SS, Brown IJ, Chan Q, Bictash M, Dumas M-E, Kochhar S, et al Metabolic profiling strategy for discovery of nutritional biomarkers: Proline betaine as a marker of citrus consumption. The American journal of clinical nutrition. 2010;92(2):436-43. https: / / doi.org/10.3945/ajcn.2010.29672

12. Guasch-Ferré M, Bhupathiraju SN, Hu FB. Use of Metabolomics in Improving Assessment of Dietary Intake. Clinical chemistry. 2018;64(1):82. https:/ / doi. org / 10.1373/ clinchem.2017.272344

13. Posma JM, Garcia-Perez I, Frost G, Aljuraiban GS, Chan Q, Van Horn L, et al. Nutriome-metabolome relationships provide insights into dietary intake and metabolism. Nat Food. 2020;1(7):426-36. https:/ / doi.org/10.1038/s43016-0200093-y

14. Loo RL, Zou X, Appel LJ, Nicholson JK, Holmes E. Characterization of metabolic responses to healthy diets and association with blood pressure: application to the Optimal Macronutrient Intake Trial for Heart Health (OmniHeart), a randomized controlled study. The American Journal of Clinical Nutrition. 2018;107(3):323-34. https:/ / doi.org/10.1093/ajcn/nqx072

15. Wilson T, Garcia-Perez I, Posma JM, Lloyd AJ, Chambers ES, Tailliart K, et al Spot and Cumulative Urine Samples Are Suitable Replacements for 24-Hour Urine Collections for Objective Measures of Dietary Exposure in Adults Using Metabolite Biomarkers. J Nutr. 2019;149(10):1692-700. https:/ / doi org $/ 10.1093 /$ jn/nxz138

16. Beckmann M, Wilson T, Lloyd AJ, Torres D, Goios A, Willis ND, et al. 
Challenges Associated With the Design and Deployment of Food Intake Urine Biomarker Technology for Assessment of Habitual Diet in Free-Living Individuals and Populations-A Perspective. Frontiers in nutrition (Lausanne). 2020;7:602515. https: / / doi.org/10.3389/ fnut.2020.602515

17. Jack CR, Therneau TM, Weigand SD, Wiste HJ, Knopman DS, Vemuri P, et al. Prevalence of Biologically vs Clinically Defined Alzheimer Spectrum Entities Using the National Institute on Aging-Alzheimer's Association Research Framework. JAMA neurology. 2019;76(10). https:/ / doi.org/10.1001/ jamaneurol.2019.1971

18. Jack CR, Jr., Bennett DA, Blennow K, Carrillo MC, Dunn B, Haeberlein SB, et al. NIA-AA Research Framework: Toward a biological definition of Alzheimer's disease. Alzheimers Dement. 2018;14(4):535-62. https://doi. org/10.1016/j.jalz.2018.02.018

19. Ahmed RM, Paterson RW, Warren JD, Zetterberg H, O'Brien JT, Fox NC, et al. Biomarkers in dementia: clinical utility and new directions. Journal of neurology, neurosurgery and psychiatry. 2014;85(12):1426-34. https:// doi. org/10.1136/jnnp-2014-307662

20. van der Lee SJ, Teunissen CE, Pool R, Shipley MI, Teumer A, Chouraki V, et al Circulating metabolites and general cognitive ability and dementia: Evidence from 11 cohort studies. Alzheimers Dement. 2018;14(6):707-22. https:/ / doi. org/10.1016/j.jalz.2017.11.012
21. Jiang Y, Zhu Z, Shi J, An Y, Zhang K, Wang Y, et al. Metabolomics in the Development and Progression of Dementia: A Systematic Review. Frontiers in neuroscience. 2019;13:343-.https: / / doi.org/10.3389/ fnins.2019.00343

22. Kurbatova N, Garg M, Whiley L, Chekmeneva E, Jiménez B, Gómez-Romero $\mathrm{M}$, et al. Urinary metabolic phenotyping for Alzheimer's disease. Sci Rep. 2020;10(1):21745. https:/ / doi.org/10.1038/s41598-020-78031-9

23. Low DY, Lefèvre-Arbogast $S$, González-Domínguez R, Urpi-Sarda M, Micheau P, Petera M, et al. Diet-Related Metabolites Associated with Cognitive Decline Revealed by Untargeted Metabolomics in a Prospective Cohort. Molecular Nutrition \& Food Research. 2019;63(18):n/a-n/a. https:/ / doi.org/10.1002/ mnfr.201900177

How to cite this article: K.A. Abbott, J.M. Posma, I. Garcia-Perez, et al. Evidence-Based Tools for Dietary Assessments in Nutrition Epidemiology Studies for Dementia Prevention. J Prev Alz Dis 2022;1(9):49-53, http:/ /dx.doi. org/10.14283/jpad.2022.6 